\title{
Factors Associated with Surgical Site Infection in Post-Cesarean Section: A Case-Control Study in a Peruvian Hospital
}

\author{
Kelly Yerba $^{1}$, Virgilio Failoc-Rojas ${ }^{2}$, Sandra Zeña-Ñañez ${ }^{3,4}$, Mario Valladares- \\ Garrido $^{4}$
}

\author{
OPEN ACCESS \\ Citation: Kelly Yerba, Virgilio Failoc- \\ Rojas, Sandra Zeña-Ñañez, Mario \\ Valladares-Garrido. Factors Associated \\ with Surgical Site Infection in Post- \\ Cesarean Section: A Case-Control Study \\ in a Peruvian Hospital. Ethiop J Health \\ Sci.2020;30(1):95. doi:http:// \\ dx.doi.org/10.4314/ejhs.v30i1.12 \\ Received: July 17, 2019 \\ Accepted: September 11, 2019 \\ Published: January 1, 2020 \\ Copyright: (C) 2020 Kelly V.Y., et al. \\ This is an open access article distributed \\ under the terms of the Creative Commons \\ Attribution License, which permits \\ unrestricted use, distribution, and \\ reproduction in any medium, provided the \\ original author and source are credited. \\ Funding: The Fogarty International \\ Center of the National Institutes of Health \\ (NIH) training under Award Number \\ D43TW001140 to SZN and U2R \\ TWOIOI 14 to VFR. The National \\ Institute of Mental Health and NIH under \\ Award Number D43TW009343 as well \\ as the University of California Global \\ Health Institute to MVG. The funders had \\ no role in study design, data analysis, \\ decision to publish, or preparation of the \\ manuscript. \\ Competing Interests: The authors \\ declare that this manuscript was approved \\ by all authors in its form and that no \\ competing interest exists. \\ Affiliation and Correspondence: \\ ${ }^{1}$ Universidad Nacional de San Agustin, \\ Arequipa, Peru \\ ${ }^{2}$ Universidad San Ignacio de Loyola, \\ Vicerrectorado de Investigación, \\ Unidad de Investigación para la \\ Generación y \\ Síntesis de Evidencias en Salud. Lima, \\ Peru \\ ${ }^{3}$ Universidad Nacional Pedro Ruiz \\ Gallo, Lambayeque, Peru \\ ${ }^{4}$ Universidad Continental, Lima, Peru \\ *Email: virgiliofr@gmail.com
}

\author{
ABSTRACT
}

BACKGROUND: Surgical site infection is the main cause of morbidity and mortality in post-cesarean section patients. The objective of this study was to determine the preoperative factors associated with the surgical site infection in patients undergoing cesarean emergency section in the Goyeneche Hospital of Arequipa.

METHODS: We conducted a cases-control study on women who presented with surgical site infection after cesarean section in a hospital in Arequipa, Peru. We evaluated associated factors to surgical site infection after cesarean section (sociodemographics and clinical variables). For the inferential analysis, we estimated logistic regression using odds ratio.

RESULTS: Out of a total of 4346 cesarean sections, only 2.4\% developed surgical site infection. The associated factors to surgical site infection were mild anemia (OR: 2.80), moderate anemia (OR: 2.71), number of vaginal examinations higher than five (OR:2.71) and onset of labor (OR: 1.95).

CONCLUSION: Patients with risk factors such as anemia, onset of labor and five or more vaginal examinations should be evaluated after surgery to prevent surgical site infection.

KEYWORDS: Surgical wound infection; post-cesarean section; associated factors (Source: MeSH-PubMed)

\section{INTRODUCTION}

Cesarean section is one of the most common procedures that is growing in all countries. Post-cesarean complications may manifest themselves as bruises, seroma or dehiscence and wound infections even as necrotizing fasciitis (1).

Surgical site infection (SSI) is the main cause of morbidity and mortality in post-cesarean section patients (2-4). As a general rule, it is considered that the onset of an SSI doubles the normal stay in hospital and costs for that operation. In the United States, it was taken into account that an SSI extends the stay in hospital to 7.3 days with an additional cost of 3,200 dollars, increasing the length of stay of a patient and the hospitalization cost $(2,5)$. 
Wound infection manifests itself with erythema and induration of the incision that usually develops 4 to 7 days after the cesarean section $(2,5)$. When the wound infection develops within 48 hours, the offending organisms are usually groups A or Betahemolytic Streptococcus. Other common pathogens that intervene in wound infections are Ureaplasma urealyticum, Staphylococcus epidermidis, Enterococcus facialis, Staphylococcus aureus, Escherichia coli and Proteus mirabilis (6).

The magnitude of the problem widely varies in the regions, but the SSI prevalence ranges from $2 \%$ to $10 \%$ on average (5-8). In addition, the favorable effect of the antibiotic prophylaxis in the reduction of infection cases associated with elective or emergency cesarean section has been proven, but in spite of that, there are still post-cesarean section surgical wounds. Based on this, it is necessary to identify preoperative factors associated with the SSI in hospitals, particularly onset of labor, duration of membrane rupture, number of internal vaginal examinations and hemoglobin level (5-8). Knowing the risk factors will allow an extended post-cesarean evaluation to the group of women. Therefore; in this study, the objective was to identify the preoperative factors associated with the SSI in patients undergoing cesarean section at Hospital Goyeneche in Arequipa, Peru.

\section{MATERIAL AND METHODS}

Study design: We conducted a case-control retrospective study assessing the factors associated with post-cesarean section wound infection at Hospital Goyeneche in Arequipa from 2015 to 2018.

Procedures: The statistics service of the hospital gave us information about the patients who underwent cesarean section during the research. It is worth mentioning that the hospital has implemented an antibiotic prophylaxis protocol (with first-generation cephalosporins) in all cases of cesarean section since 2014. The inclusion criteria in the case study were pregnant patients who underwent cesarean section and were diagnosed with SSI (by bacteriological culture of wound secretion) within 30 days of emergency obstetric surgery, and who had been administered antibiotic prophylaxis. Control patients were determined after the inclusion of case-patients and met the following inclusion criteria: similar age $( \pm$ 2 years), cesarean section, surgery conducted on the same day, without a history of post-cesarean section infectious complication within 30 days, and who had been administered antibiotic prophylaxis. In addition, patients diagnosed with diabetes mellitus, immunodeficiency, malignancies and any type of immunosuppression were excluded.

The sample was calculated by the frequency of variable premature rupture of membranes, evaluated in a previous study (9), to contrast hypotheses in independent groups in case and control studies; the variable premature rupture of membranes was considered with an exposed proportion of $34 \%$ and an OR to calculate of 3.5 . For each case, four controls were taken by matching age and date of cesarean section, giving a total of 35 cases and a total of 140 controls of women without post-cesarean section infection, selected through a sampling of a database with patients who met such conditions.

Data collection and processing: The clinical records were reviewed to collect sociodemographic data (age, marital status, education level, occupation), clinical data (body mass index, hemoglobin level, comorbidity, and postoperative diagnosis), obstetric data (parity, gestational age), delivery duration, duration of membrane rupture, number of internal vaginal examinations and length of stay in hospital. The indicator of hemoglobin level $(\mathrm{Hb})$ was classified according to the World Health Organization guidelines for pregnant women at the beginning of the third quarter, and due to the location of Arequipa at 2,400 masl, and for that reason, a value less than 12.3 was considered as anemia.

Statistical analysis: For the descriptive analysis of numerical variables, normality assumptions were evaluated using analytical parameters (means, medians and kurtosis) and histogram. The categorical variables were described using samples and percentages. We evaluated the association between operative wound infection after cesarean and categorical co-variables of interest using Chisquared test. Student's t for independent samples was used to compare the means between the groups prior to normality test. We consider the level of significance at 5\%. For simple and multiple regression, we used logistic regression to calculate 
odds ratios (ORs) and 95\% confidence intervals (95\% CIs). For multiple regression, the model was adjusted for associated variables in the simple regression. The statistical analyses were conducted using STATA version 12.0.

Ethical considerations: To carry out this study, the authorization at the Hospital Goyeneche of Arequipa was obtained. The research study met the fundamental principles of the declaration of Helsinki: respect to personal dignity, justice and charity; no names of patients were saved, thus maintaining anonymous database.

\section{RESULTS}

A total of 4,346 cesarean sections were recorded for 3 years; only 106(2.4\%) patients diagnosed with post-cesarean section SSI were recorded. The pathogen most frequently cultivated was Bhemolytic Streptococcus. Based on the histories selected, 35 were cases and 140 controls, giving a total of 175 patients during the 3 years of study. The mean age was 27, and the other sociodemographic characteristics were similar for both groups (Table 1).

Table 1: Clinical and sociodemographic characteristics of patients with SSI after cesarean section in a peruvian hospital.

\begin{tabular}{lllll}
\hline CHARCTERISTICS & CASES & CONTROLS & \multicolumn{2}{l}{ TOTAL } \\
\hline & $\mathrm{N}=35$ & $\mathrm{~N}=140$ & $\mathrm{~N}^{\circ}$ & $\%$ \\
\hline Maternal age* & 25 & 28 & 27 & {$[16-42]$} \\
Marital status & & & & \\
Single & $3(8.57 \%)$ & $13(9.29 \%)$ & 16 & 9.14 \\
Married & $5(14.29 \%)$ & $20(14.29 \%)$ & 25 & 14.29 \\
Cohabitant & $27(77.14 \%)$ & $107(76.43 \%)$ & 134 & 76.57 \\
Education level & & & & \\
Primary & $3(8.57 \%)$ & $5(3.57 \%)$ & 8 & 4.57 \\
Secondary & $24(68.57 \%)$ & $122(87.14 \%)$ & 146 & 83.43 \\
Higher education & $8(22.86 \%)$ & $13(9.29 \%)$ & 21 & 12 \\
Urinary tract infection & $3(8.57 \%)$ & $7(5 \%)$ & 10 & 5.71 \\
More than 5 VEs & $30(85.71 \%)$ & $77(55 \%)$ & 107 & 61.14 \\
Onset of labor & $13(37.14 \%)$ & $16(11.43 \%)$ & 29 & 16.57 \\
PROM & $8(22.86 \%)$ & $13(9.29 \%)$ & 21 & 12 \\
Weight* & $67.5[51-93]$ & $69[47-95.5]$ & 69 & {$[47-95.5]$} \\
BMI** & $29.91 \pm 3.14$ & $30.88 \pm 3.56$ & 30,69 & \pm 3.49 \\
Gestational age* & $39[33-41]$ & $39[38-41]$ & 39 & {$[33-41]$} \\
Hemoglobin* & $9.86 \pm 0.93$ & $10.43 \pm 1.37$ & 10.52 & {$[5.42-13.02]$} \\
Anemia & & & & \\
$\quad$ No & $4(11.43 \%)$ & $49(35 \%)$ & 53 & 30.29 \\
$\quad$ Mild anemia & $14(40 \%)$ & $50(35.71 \%)$ & 64 & 36.57 \\
Moderate anemia & $17(48.57 \%)$ & $41(29.29 \%)$ & 58 & 33.14 \\
\hline The & & & & \\
\end{tabular}

*The mean was used; **The median was used; PROM: Premature Rupture of Membranes

In the analysis of the adjusted model, it was estimated that the odds of developing SSI in the group that had more than five VEs is 2.71 times the odds of presenting SSI in those who did not have

DOI: http://dx.doi.org/10.4314/ejhs.v30i1.12 
this condition (OR: 2.71, 95\% CI: 1.07-6.82). Also, women with onset of labor (OR: $1.95,95 \% \mathrm{CI}$ : 1.11-3.43), mild anemia (OR: 2.80, 95\% CI: 1.03 -
7.64) and moderate anemia (OR: $2.71,95 \% \mathrm{CI}$ : 1.00-7.31) had more chances of developing SSI after cesarean section (Table 2).

Table 2: Factors associated with SSI after cesarean section in a peruvian hospital

\begin{tabular}{|c|c|c|c|c|}
\hline CHARACTERISTICS & $\begin{array}{l}\text { Crude model } \\
\text { cOR }(95 \% \text { CI })\end{array}$ & $P$ & $\begin{array}{l}\text { Adjusted model } \\
\text { aOR } \$(95 \% \text { CI })\end{array}$ & $p$ \\
\hline Maternal age* & $0.92(0.86-0.98)$ & 0.015 & $0.95(0.89-1.01)$ & 0.083 \\
\hline More than five VEs & $3.81(1.55-9.34)$ & 0.003 & $2.71(1.07-6.82)$ & 0.034 \\
\hline Onset of labor & $2.97(1.70-5.19)$ & 0.001 & $1.95(1.11-3.43)$ & 0.019 \\
\hline Membrane rupture & $2.17(0.57-4.19)$ & 0.001 & $1.13(0.62-2.06)$ & 0.685 \\
\hline Weight* & $0.98(0.95-1.02)$ & 0.532 & Not included + & \\
\hline BMI** & $0.93(0.84-1.03)$ & 0.192 & Not included + & \\
\hline Gestational age* & $1.20(0.89-1.61)$ & 0.229 & Not included + & \\
\hline Anemia* & $0.79(0.63-0.98)$ & 0.041 & Not included + & \\
\hline $\begin{array}{l}\text { No } \\
\text { Mild anemia } \\
\text { Moderate anemia }\end{array}$ & $\begin{array}{l}2.89(1.01-8.28) \\
3.88(1.39-10.80)\end{array}$ & $\begin{array}{l}1 \\
0.047 \\
0.009\end{array}$ & $\begin{array}{l}2.80(1,03-7,64) \\
2.71(1,00-7,31)\end{array}$ & $\begin{array}{l}1 \\
0.043 \\
0.048\end{array}$ \\
\hline Marital status & & & & \\
\hline Single & & 1 & Not included + & \\
\hline Married & $1.06(0.29-3.86)$ & 0.922 & & \\
\hline Cohabitant & $1.07(0.36-3.14)$ & 0.896 & & \\
\hline \multicolumn{5}{|l|}{ Education level } \\
\hline Primary & & 1 & Not included + & \\
\hline Secondary & $0.43(0.16-1.15)$ & 0.094 & & \\
\hline Higher education & $1.01(0.35-2.89)$ & 0.976 & & \\
\hline \multicolumn{5}{|l|}{ Occupation } \\
\hline Student & & 1 & Not included + & \\
\hline Housewife & $0.38(0.13-1.07)$ & 0.060 & & \\
\hline Office & $0.41(0.12-1.39)$ & 0.153 & & \\
\hline Urinary tract infection & $1.54(0.57-4.19)$ & 0.391 & Not included + & \\
\hline
\end{tabular}

VE: Vaginal examinations; *The mean was used; $\$$ The linear models with logistic regression and binomial family functions adjusted to the variables presented were used. + Only variables with likelihood ratio test lower than 0.10 were only included in the crude analysis.

\section{DISCUSSION}

We found a SSI after cesarean prevalence of $2.4 \%$, similar frequency to other studies described in Brazil (9) where there was a prevalence of $1.44 \%$. But slightly lower than other studies in Ethiopia and Nigeria, where the prevalence was $6.8 \%$ and $11 \%$ respectively $(8,10)$. Other studies such as in the USA (11) found a prevalence of 5.5\%. This can be due to the fact that this study included patients who received antibiotic prophylactic treatment (first-generation cephalosporin), and, excluded patients with comorbidity such as mellitus diabetes, immunodeficiency, and malignancies.

It is important to highlight that the antibiotic prophylaxis prior to surgery continues to be a much-discussed topic and as we know, is part of one of the operative stages $(12,13)$. So, it is 
suggested the use of cefazolin 1 gr or azithromycin $500 \mathrm{mg}$ before the cesarean section (14). This bias has been eliminated by including in the control group only patients without history of infection or the use of antibiotics until 30th postpartum day, apart from prophylaxis. Risk factors well documented in the literature, such as emergency cesarean section, duration of ruptured membranes, duration of surgery, excessive vaginal manipulation, obesity, and surgical risk must be analyzed for prophylaxis antibiotic treatment (713).

This study demonstrated an association between post-cesarean section SSI and the number of internal vaginal examinations higher than 5 with an OR of 2.71. These results are similar with studies conducted in other regions $(9,10)$, and can be explained by the possible transmission of microorganisms to surface areas with vaginal examinations.

The time of membrane rupture had an cOR of 2.17 in the crude model. Some studies established as a cut-off point a time longer than 12 hours, and this has a high-risk factor for SSI $(1,8,10)$. When the membranes are ruptured, the amniotic fluid is no longer sterile and can act as a means of transport, the bacteria can come into contact with the tuterus and the incisions of the skin, which causes the risk of developing infections in the surgical site (9).

The onset of labor had an a aOR of 1.95. Some studies established as a cut-off point a time longer than one hour for SSI risk. Some authors consider that prolonged labor and the rupture of membranes contribute to the colonization of the amniotic fluid from the normal flora of the lower genital tract and lead to the surgical wound and contamination of the peritoneal cavity (8). This is supported by other studies $(8,10)$.

As for the anemia, it was observed that a higher level of hemoglobin is associated with a lower risk of having SSI. It is also observed that having suffered from moderate anemia was a highrisk factor for SSI with an ORc 2.71. This explains why the hemoglobin reduces the oxygen tension and why there is less collagen synthesis. This increasing the risk of wound infection by compromising the activity of the macrophages and preventing the progress of the wound healing. Since it produces a less stable scar and favors dehiscence and infection (15). And this is supported in other studies $(8,10)$.

Our research has some limitations. The first is Berkson's probable bias because we selected cases from a single hospital; the risk factors found are specific to that center. This is due to the fact that the Goyoneche Hospital has a level III of complexity, and it is possible that a high amount of care and referrals for cesarean section are registered. Therefore, it is not possible to generalize our findings to the entire study population. Finally, there was a probable measurement bias since the variables considered as potential risk factors were obtained from the hospital's clinical history. Also, the postoperative risk factors such as wound care, cleaning, etc. cannot be controlled. However, we believe that the factors found are important to expand future research.

We recommend future studies where the evaluation is extended to various hospitals. The recovery from cesarean section is very difficult for women who develop postoperative infections. These infections can affect her pelvic organs, surgical wound, and respiratory and urinary tracts. This makes us think that healthy practices should be promoted in patients and in personnel who will conduct the cesarean section. We conclude that anemia, premature rupture of membranes, onset of labor and 5 or more vaginal examinations were preoperative factors associated with development of SSI. Patients with these risk factors should be evaluated after surgery to prevent SSI.

\section{REFERENCES}

1. Kawakita T, Landy HJ. Surgical site infections after cesarean delivery: epidemiology, prevention and treatment. Matern Health Neonatol Perinatol. 2017; 5:3-12.

2. Olsen MA, Butler AM, Willers DM, Devkota P, Gross GA, Fraser VJ. Risk factors for surgical site infection after low transverse cesarean section. Infect Control Hosp Epidemiol. 2008; 29:477-84. 
3. Santalla A, López-Criado MS, Ruiz MD, Fernández-Parra J, Galloa JL, Montoya F. Surgical site infection. Prevention and treatment. Clin Invest Ginecol Obstet 2007; 34:189-96.

4. Chang NVF, Dalmau NMB, Rodriguez LAM, Frias NL, Leyva LM. Infection of the postcesarean section surgical site. Medisan.2016;20(5)

5. Blumenfeld YJ, El-Sayed YY, Lyell DJ, Nelson LM, Butwick AJ. Risk factors for prolonged postpartum length of stay following cesarean delivery. Am J Perinatol. 2015; 32(9):825-832.

6. Martens MG, Kolrud BL, Faro S, Maccato M, Hammill H. Development of wound infection or separation after cesarean delivery. Prospective evaluation of 2,431 cases. $J$ Reprod Med. 1995;40:171-175.

7. Moreira de Lima D, Loewen Wall D, Albimara H, Falcade A, De Morais Chaves A, Rocha de Souza M. Fatores de riscos para infecção no puerpério cirúrgico, Cogitare Enferm. 2014; 19(4):734-40

8. Wodajo S, Belayneh M, Gebremedhin S. Magnitude and Factors Associated with PostCesarean Surgical Site Infection at Hawassa University Teaching and Referral Hospital, Southern Ethiopia: A Cross-sectional Study. Ethiop J Health Sci. 2017 May;27(3):283-290.
9. Farret TC, Dallé J, Monteiro Vda S, Riche $\mathrm{CV}$, Antonello VS. Risk factors for surgical site infection following cesarean section in a Brazilian Women's Hospital: a case-control study. Braz J Infect Dis. 2015;19(2):113-7.

10. Gelaw KA, Aweke AM, Astawesegn FH, Demissie BW, Zeleke LB. Surgical site infection and its associated factors following cesarean section: a cross sectional study from a public hospital in Ethiopia. Patient Saf Surg. $2017 ; 12 ; 11: 18$.

11. Moulton LJ, Munoz JL, Lachiewicz M, Liu X, Goje O. Surgical site infection after cesarean delivery: incidence and risk factors at a US academic institution. J Matern Fetal Neonatal Med. 2018; 31(14):1873-1880.

12. ACOG Practice Bulletin No. 120: Use of prophylactic antibiotics in labor and delivery. Obstetrics and gynecology. 2011;117(6):147283.

13. Tita AT, Szychowski JM, Boggess K, et al. Adjunctive Azithromycin prophylaxis for cesarean delivery. $N$ Engl $J$ Med. 2016;375(13):1231-1241

14. Baaqeel H,Baaqeel R. Timing of administration of prophylactic antibiotics for caesarean section: a systematic review and meta-analysis. BJOG.2013. 120. 661-669

15. Gordillo GM, Sen CK. Revisiting the essential role of oxygen in wound healing. Am J Surg. 2003;186:259-26. 